Check for updates

Cite this: Mater. Adv., 2021, 2, 3020

Received 2nd February 2021, Accepted 8th March 2021

DOI: 10.1039/d1ma00097g

rsc.li/materials-advances

\section{An efficient photo-chemo combination therapeutic platform based on targeted reduction-responsive self-crosslinked polymer nanocapsules $\dagger$}

\author{
Shengbei Qi, ${ }^{a}$ Chaoyong Liu, ${ }^{\mathrm{b}}$ Meng Qin, ${ }^{\mathrm{b}}$ Cheng Tao, ${ }^{\mathrm{a}}$ Jie Liu, ${ }^{\mathrm{a}}$ Yuan Le, ${ }^{a}$ \\ Jiexin Wang, (D) *ab Jianjun Zhang (D) *a and Yunfeng Lu (D) ${ }^{c}$
}

\begin{abstract}
Photo-chemo combination therapy holds great promises for cancer treatment; thus developing efficient approaches for delivering photo-chemo combination reagents has long been pursued. Herein, an amphiphilic branched copolymer, four-arm poly(ethylene glycol)-poly(propylene sulfide)-cyclic(Arg-GlyAsp-D-Phe-Lys) (PEG-PPS-CRGD), was synthesized to prepare targeted self-crosslinked polymer nanocapsules consisting of a disulfide bond and the $\alpha_{v} \beta_{3}$ integrin-targeting ligand CRGD. Furthermore, by coloading both indocyanine green (ICG) and doxorubicin (DOX) in PEG-PPS-CRGD nanocapsules, a high-performance photo-chemo combination therapeutic platform designated as DINCs was successfully developed. The dissolution rate investigations demonstrated that DINCs exhibited sensitive reduction-responsive drug release profiles during the incubation with glutathione (GSH), while the drug release rate could be further accelerated by NIR laser irradiation. The in vitro studies showed that DINCs had outstanding cellular uptake, photothermal effects and ROS generation under laser irradiation, which significantly enhanced cytotoxicity in the treatment of squamous cells (SCC-15). Moreover, the in vivo results showed that synergistic photo-chemo combination treatment by DINCs plus laser irradiation could completely eliminate the tumor of squamous cell carcinoma with favorable biological safety.
\end{abstract}

\section{Introduction}

Phototherapy, including photothermal therapy (PTT) and photodynamic therapy (PDT), has attracted enormous attention in cancer treatment. ${ }^{1-5}$ Notably, since single chemotherapy cannot achieve satisfactory treatment effects due to the inefficient tumor killing activity, the combination of phototherapy and chemotherapy is considered to be a very promising strategy to enhance the effectiveness of cancer therapies. ${ }^{6-10}$ In addition to directly killing cancer cells by hyperthermia and reactive oxygen species (ROS), phototherapy can also promote therapeutic molecule internalization into cells by the "photochemical internalization" (PCI) effect, resulting in reduced side effects and enhanced therapeutic effects. ${ }^{11-14}$ To date, although various

\footnotetext{
${ }^{a}$ College of Chemical Engineering, Beijing University of Chemical Technology, Beijing 100029, P. R. China. E-mail: zhangij@mail.buct.edu.cn

${ }^{b}$ Beijing Advanced Innovation Center for Soft Matter Science and Engineering, Beijing University of Chemical Technology, Beijing 100029, P. R. China. E-mail:wangjx@mail.buct.edu

${ }^{c}$ Department of Chemical and Biomolecular Engineering, University of California at Los Angeles, Los Angeles 90095, USA

$\dagger$ Electronic supplementary information (ESI) available. See DOI: 10.1039/ d1ma00097g
}

delivery approaches have been established for delivering photochemo combination reagents, the potential applications of these delivery systems are hampered by the poor structural stability, and lack of stimulus-responsive ability.

Covalently bonded polymer nanocarriers named as nanogels or nanocapusles hold great prospects for targeted drug delivery because they can stably transport therapeutic agents to the disease location with minimal premature leakage, and controllably activate them by both endogenous and exogenous stimulus factors, such as acidity, redox substances, enzymes, and light, to achieve an optimal efficacy. ${ }^{15-20}$ Owing to the heterogeneous redox potential gradient in malignant tumor tissues, such as glutathione (GSH) in the cytoplasm, polymer nanocarriers containing disulfide bonds have been extensively explored for fabricating reduction-responsive drug delivery systems. ${ }^{21-25}$ In particular, the bonding reaction of the disulfide bond between thiols has mild and friendly reaction conditions, which is an easy-to-accomplish process.

Over the past decade, poly(ethylene glycol) (PEG) has been widely utilized for fabricating drug nanocarriers due to its outstanding aqueous solubility, non-toxicity, non-immunogenicity, and electric neutrality, which significantly improved drug stability, blood circulation times, and tumor-targeting ability. ${ }^{26-31}$ 
As compared to traditional linear PEG, branched multi-arm PEG has a higher density of polymer chains that could achieve better protection for the loaded drugs in the treatment. ${ }^{32}$ Otherwise, multi-arm PEG provides more sites of end groups, which can be useful for modification of different functional units for constructing high-performance drug nanocarriers. ${ }^{33-35}$

Herein, we designed a four-arm poly(ethylene glycol)-poly (propylene sulfide)-cyclic(Arg-Gly-Asp-D-Phe-Lys) (PEG-PPS-cRGD) amphiphilic branched copolymer with end-capping pyridine dithione groups, and used it to successfully develop a photochemo combination therapy platform by incorporating both doxorubicin (DOX) and indocyanine green (ICG) into a single PEG-PPS-cRGD nanocapsule for targeted treatment of squamous cell carcinoma. As shown in Fig. 1, first, DOX and ICG were encapsulated into PEG-PPS-cRGD by a single-step solvent-antisolvent method to form DOX and ICG-coloaded PEG-PPS-cRGD nanocapsules (named DINCs), which were cross-linked via a disulfide bond through a disulfide exchange reaction under triethylamine (TEA) initiation). The PEG component can improve the stability and blood circulation time of DINCs, making it effective for accumulation in tumor sites through the enhanced permeability and retention (EPR) effect. Subsequently, the cRGD component can assist DINC bonding on the surface of squamous cells (SCC-15 cell-line) via $\alpha_{v} \beta_{3}$ integrin and its further entry into the cells by the $\alpha_{v} \beta_{3}$-mediated endocytosis pathway and PCI effect under short-time NIR light irradiation. Furthermore, long-term NIR light irradiation was utilized to activate DINCs to increase the temperature and produce ${ }^{1} \mathrm{O}_{2}$, which can not only induce cell death but also promote the release of combined DOX by enhancing the drug solubility. Moreover, the high level of GSH in the cytoplasm can also accelerate DOX release by breaking the disulfide bonds in DINCs. Finally, the free DOX will spread into

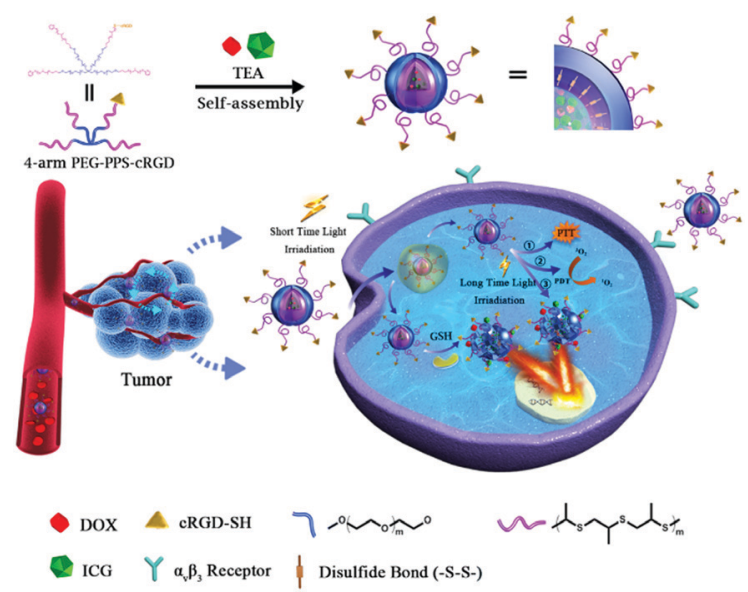

Fig. 1 Schematic illustration of the preparation procedure and functional mechanism of DINCs. I, self-crosslinked DINCs were prepared via a solvent-antisolvent process under initiation of TEA; II, DINCs target cancer cells via the $\alpha_{v} \beta_{3}$-integrin receptor; III, DINCs internalize into cells by the receptor-mediated endocytosis pathway and $\mathrm{PCl}$ effect initiated under short-term light irradiation; IV, DINCs achieve PTT, PDT and release of DOX cargo under long-term light irradiation; $\mathrm{V}$, DINCs can also significantly release DOX cargo by the high-level of GSH in cytoplasm; VI, released DOX enters the nucleus and plays a chemotherapeutic role by intercalation in DNA. the cell nucleus to play a role in chemotherapy. This photo-chemo combination therapeutic platform provides a promising strategy for enhancing anticancer efficacy.

\section{Experimental section}

\section{Materials}

Indocyanine green (ICG, 75\%) was purchased from Shanghai Macklin Biochemical Co., Ltd (China). 1,3-Diphenylisobenzofuran (DPBF), doxorubicin hydrochloride (DOX.HCl) and $2^{\prime}, 7^{\prime}$ dichlorodihydro-fluorescein diacetate (DCFH-DA) were purchased from Aladdin (China). 3-(4,5-Dimethylthiazol-2-yl)-2,5-diphenyltetrazolium bromide (MTT) was purchased from Sigma-Aldrich (USA). Calcein-AM/propidium iodide (PI) double stain kit, 4',6diamidino-2-phenylindole (DAPI) and Annexin V-FITC/PI apoptosis detection kit were obtained from Shanghai Beyotime Biochemical Co., Ltd (China). High glucose Dulbecco's modified Eagle's medium (DMEM), fetal bovine serum and penicillin/streptomycin were bought from Thermo Fisher Scientific (USA). SCC-15 cells (human squamous cell carcinoma cell line) were obtained from American Type Culture Collection (ATCC, USA).

\section{Preparation and characterization of DINCs}

The four-arm PEG-PPS-cRGD copolymer was synthesized via three steps according to our previous work. ${ }^{36}$ Furthermore, $4 \mathrm{mg}$ of ICG, $2 \mathrm{mg}$ of DOX. $\mathrm{HCl}$ and $4.5 \mu \mathrm{L}$ of triethylamine (TEA) were dissolved in $0.4 \mathrm{~mL}$ of DMSO and mixed for $15 \mathrm{~min}$ with stirring. Additionally, $4 \mathrm{~mL}$ of PEG-PPS-cRGD aqueous solution (0.5 wt\%) was slowly added to the ICG and DOX solution. After addition of PEG-PPS-cRGD, the reaction solution was stirred at $45{ }^{\circ} \mathrm{C}$ for another $2 \mathrm{~h}$. Finally, the products were dialyzed against ultrapure water through a dialysis bag (molecular cut-off: $3500 \mathrm{Da}$ ) to remove unloaded DOX, ICG and DMSO. The target product was then obtained by lyophilizing the solution at $-55{ }^{\circ} \mathrm{C}$ for $72 \mathrm{~h}$.

The typical structure of the nanocapsules was investigated by transmission electron microscopy (TEM, Hitachi HT7700, Japan). The size distribution of the nanocapsules was determined using dynamic light scattering (DLS, Malvern Nano $\mathrm{ZS}, \mathrm{UK}$ ), reported as the $Z$-average (intensity-based mean particle diameter). The DOX and ICG loading capacity (LC) and entrapment efficiency (EE) of the DINCs were measured using a UVvisible spectrophotometer (Varian Cary 50, USA) with absorption wavelengths at $488 \mathrm{~nm}$ and $633 \mathrm{~nm}$ for DOX and ICG, respectively, and calculated according to the following formula:

$$
\begin{aligned}
& \text { LC }(\text { wt } \%)=(\text { weight of loaded drug/weight of drug-loaded } \\
& \text { nanocapsules) } \times 100 \% \text {. }
\end{aligned}
$$

$\mathrm{EE}(\mathrm{wt} \%)=($ weight of loaded drug/weight of initially added drug) $\times 100 \%$.

\section{Photothermal effect of DINCs}

To evaluate the photothermal efficiency of the nanocapsules, we used an infrared thermal imager (Fluke TiS20, USA) to monitor temperature changes under laser irradiation. PBS buffer, free 
ICG solution and DINC solution (ICG concentration of $14.4 \mu \mathrm{g} \mathrm{mL} \mathrm{m}^{-1}$ ) in a tube were irradiated by a $785 \mathrm{~nm}$ continuous-wave NIR laser (Beijing NBeT Group Corp, China) with an output power of $2.0 \mathrm{~W} \mathrm{~cm}^{-2}$. The temperature was also recorded using a digital thermometer every $30 \mathrm{~s}$ during the irradiation period.

\section{Detection of single oxygen detection $\left({ }^{1} \mathrm{O}_{2}\right)$ of DINCs}

The generation of ${ }^{1} \mathrm{O}_{2}$ was measured via the method reported by Wöhrle et al. Briefly, $1 \mathrm{~mL}$ of PBS buffer, free ICG solution and DINC solution (ICG concentration of $14.4 \mu \mathrm{g} \mathrm{mL}^{-1}$ ) were mixed with $100 \mu \mathrm{L}$ of DPBF $\left(0.5 \mathrm{mg} \mathrm{mL}^{-1}\right.$, freshly prepared in acetonitrile) and then diluted with acetonitrile to a final volume of $3 \mathrm{~mL}$. After vortexing for $10 \mathrm{~s}$, the mixture solutions were stirred and irradiated for $5 \mathrm{~min}\left(785 \mathrm{~nm}, 2.0 \mathrm{~W} \mathrm{~cm}^{-2}\right)$. The generation of ${ }^{1} \mathrm{O}_{2}$ accompanying the absorbance decrease in DPBF was detected at $410 \mathrm{~nm}$.

\section{In vitro DOX release from DINCs}

Two milligrams of DINCs were dispersed in $2 \mathrm{~mL}$ of PBS buffer and transferred into a dialysis bag (molecular cut-off: $3500 \mathrm{Da}$ ). The dialysis bag was then placed in $20 \mathrm{~mL}$ of PBS buffer containing $10 \mathrm{mM}$ of GSH. For laser irradiation of the samples, the dialysis bag was placed in $20 \mathrm{~mL}$ of PBS buffer or $20 \mathrm{~mL}$ of PBS buffer containing $10 \mathrm{mM}$ of GSH, and the samples were then irradiated with an NIR laser $(785 \mathrm{~nm}, 2.0 \mathrm{~W})$ for $5 \mathrm{~min}$ at the initial time. Subsequently, the samples were incubated at $37{ }^{\circ} \mathrm{C}$ for $24 \mathrm{~h}$, and the cumulative release rate of DOX from the DINCs was calculated by determining the UV absorption of DOX at $488 \mathrm{~nm}$.

\section{Cell culture and tumor model}

The human squamous cell carcinoma cell line (SCC-15 cells) was cultured in DMEM (high glucose) with 10\% fetal bovine serum (FBS) at $37{ }^{\circ} \mathrm{C}$ with $5 \% \mathrm{CO}_{2}$. Female BALB/c nude mice were obtained from Beijing Vital River Laboratories. SCC-15 tumors were created by a subcutaneous injection of $2 \times 10^{6}$ SCC-15 cells into each mouse. All the animal studies were performed in accordance with the Guidelines for Care and Use of Laboratory Animals of Beijing Vital River Laboratory Animal Technology Co., Ltd and approved by the Institutional Animal Care and Use Committee of Beijing Vital River Laboratory Animal Technology Co., Ltd.

\section{In vitro cellular uptake of DINCs}

SCC-15 cells $\left(8 \times 10^{3}\right.$ cells per well $)$ were seeded into 8 -well chambered cover glasses (Lab-Tek, Nunc, USA) in $200 \mu \mathrm{L}$ of complete DMEM and cultured overnight. The cells were treated with the designated formulations (final DOX concentration of $3.6 \mu \mathrm{g} \mathrm{mL} \mathrm{mL}^{-1}$ and ICG concentration of $14.4 \mu \mathrm{g} \mathrm{mL}^{-1}$ ) with or without short-term laser irradiation $\left(785 \mathrm{~nm}, 2.0 \mathrm{~W} \mathrm{~cm} \mathrm{~cm}^{-2}\right.$, $1 \mathrm{~min}$ ) and further incubated for another $4 \mathrm{~h}$. Then, the cells were washed 3 times with PBS and fixed with $4 \%$ paraformaldehyde $(100 \mu \mathrm{L})$ for $20 \mathrm{~min}$. The cells were then washed with PBS and stained with DAPI $\left(5 \mu \mathrm{g} \mathrm{mL} \mathrm{m}^{-1}, 100 \mu \mathrm{L}\right)$ for $15 \mathrm{~min}$ in the dark. Finally, the cells were washed at least 3 times and observed by confocal laser scanning microscopy (CLSM, Leica
TCS SP8, Germany) (DAPI, 360/460 nm; DOX, 488/515 nm; ICG, $633 / 780 \mathrm{~nm})$.

For flow cytometry, SCC-15 cells $\left(1 \times 10^{6}\right.$ cells per well $)$ were seeded into a six-well plate and cultured overnight. The cells were then treated with various designed formulations (final DOX concentration of $3.6 \mu \mathrm{g} \mathrm{mL}^{-1}$ and ICG concentration of $\left.14.4 \mu \mathrm{g} \mathrm{mL}^{-1}\right)$ with or without laser irradiation $(785 \mathrm{~nm}$, $2.0 \mathrm{~W} \mathrm{~cm}^{-2}$, and $1 \mathrm{~min}$ ) and incubated for another $4 \mathrm{~h}$. Next, the cells were harvested, detected, and quantified using a flow cytometer (FCM, Beckman Coulter MoFloXDP, USA).

\section{Detection of intracellular ROS generation of DINCs}

SCC-15 cells $\left(8 \times 10^{3}\right.$ cells per well $)$ were seeded into 8-well chambered cover glasses in $200 \mu \mathrm{L}$ of complete DMEM and cultured overnight. The cells were then treated with the designated formulations (final DOX concentration of $3.6 \mu \mathrm{g} \mathrm{mL} \mathrm{m}^{-1}$ and ICG concentration of $14.4 \mu \mathrm{g} \mathrm{mL}{ }^{-1}$ ) for $4 \mathrm{~h}$. After washing, the cells were stained with DAPI $\left(5 \mu \mathrm{g} \mathrm{mL} \mathrm{m}^{-1}, 100 \mu \mathrm{L}\right)$ for $15 \mathrm{~min}$ and DCFH-DA $(10 \mu \mathrm{M}, 100 \mu \mathrm{L})$ for $30 \mathrm{~min}$ in the dark. Eventually, the cells were washed 3 times and irradiated $\left(785 \mathrm{~nm}, 2.0 \mathrm{~W} \mathrm{~cm} \mathrm{~cm}^{-2}\right.$, $1 \mathrm{~min}$ ). Intracellular ROS images were simultaneously observed by CLSM (DAPI, 360/460 nm; DCF, 488/525 nm).

Furthermore, SCC-15 cells $\left(1 \times 10^{6}\right.$ cells per well $)$ were seeded into a six-well plate and cultured overnight. The cells were then treated with various designated formulations (final DOX concentration of $3.6 \mu \mathrm{g} \mathrm{mL} \mathrm{m}^{-1}$ and ICG concentration of $14.4 \mu \mathrm{g} \mathrm{mL}^{-1}$ ) for $4 \mathrm{~h}$. After washing, the cells were stained with DCFH-DA $(10 \mu \mathrm{M}, 1 \mathrm{~mL})$ for $30 \mathrm{~min}$ in the dark. Next, the cell medium was replaced, and laser irradiation $\left(785 \mathrm{~nm}, 2.0 \mathrm{~W} \mathrm{~cm}^{-2}\right.$, and $1 \mathrm{~min}$ ) was applied to the samples. Finally, the cells were harvested, detected, and quantified using FCM.

\section{In vitro cytotoxicity evaluation of DINCs}

The MTT assay was used to detect the cytotoxicity of PEG-PPScRGD copolymers and DINCs. SCC- 15 cells $\left(4 \times 10^{3}\right.$ cells per well $)$ were plated in a 96-well plate in $100 \mu \mathrm{L}$ of complete DMEM and cultured overnight. Then, various concentrations of PEG-PPScRGD, free DOX, and DINCs were added to the cell plate. The polymer concentrations of PEG-PPS-cRGD copolymers ranged from 0 to $360 \mu \mathrm{g} \mathrm{mL}{ }^{-1}$. The free DOX and DINCs were diluted in PBS with final DOX concentrations of 0 to $18 \mu \mathrm{g} \mathrm{mL} \mathrm{mL}^{-1}$ and final ICG concentrations of 0 to $72 \mu \mathrm{g} \mathrm{mL} \mathrm{m}^{-1}$. After a $4 \mathrm{~h}$ incubation, the cell medium was replaced, and long-term laser irradiation $(785 \mathrm{~nm}$, $2.0 \mathrm{~W} \mathrm{~cm}^{-2}, 5 \mathrm{~min}$ ) was applied to the DINC group. The cells were further incubated for another $24 \mathrm{~h}$, and $20 \mu \mathrm{L}$ of MTT solution (5 $\mathrm{mg} \mathrm{mL}^{-1}$ ) was then introduced into the cell medium for an additional $4 \mathrm{~h}$ incubation. Finally, the supernatant was removed completely, and $100 \mu \mathrm{L}$ of DMSO was added to dissolve the blue formazan crystals. The absorbance of the solution was detected using a microplate reader (Thermo Fisher, MK3, America) at $570 \mathrm{~nm}$. Cell viability was assessed via the following formula:

$$
\text { Cell viability }(\%)=A_{\text {sample }} / A_{\text {control }} \times 100 \%
$$

For the in vitro live-dead assay, SCC-15 cells $\left(8 \times 10^{3}\right.$ cells per well) were seeded into 8-well chambered cover glasses in $200 \mu \mathrm{L}$ 
of complete DMEM and cultured overnight. Then, the cells were incubated with PBS, free DOX $\left(7.2 \mu \mathrm{g} \mathrm{mL} \mathrm{m}^{-1}\right)$ and DINCs (containing $7.2 \mu \mathrm{g} \mathrm{mL} \mathrm{m}^{-1}$ of DOX and $28.8 \mu \mathrm{g} \mathrm{mL}^{-1}$ of ICG) for $4 \mathrm{~h}$. After washing the cells, long-term laser irradiation $(785 \mathrm{~nm}$, $2.0 \mathrm{~W} \mathrm{~cm}^{-2}$, and $5 \mathrm{~min}$ ) was applied to the DINC group, and the cells were further cultured for another $24 \mathrm{~h}$. Afterwards, the cells were washed 3 times with PBS and further stained with $100 \mu \mathrm{L}$ of complete DMEM containing calcein-AM and PI according to the manufacturer's protocol. Finally, the stained cells were imaged via CLSM (calcein-AM, 495/515 nm; PI, 535/ $617 \mathrm{~nm})$.

For the in vitro apoptosis assay, SCC-15 cells $\left(1 \times 10^{6}\right.$ cells per well) were seeded into a six-well plate and cultured overnight. Then, the cells were incubated with PBS, free DOX (7.2 $\mu \mathrm{g} \mathrm{mL} \mathrm{mL}^{-1}$ ) and DINCs (containing $7.2 \mu \mathrm{g} \mathrm{mL}{ }^{-1}$ of DOX and $28.8 \mu \mathrm{g} \mathrm{mL} \mathrm{mL}^{-1}$ of ICG) for $4 \mathrm{~h}$. After washing the cells, longterm laser irradiation $\left(785 \mathrm{~nm}, 2.0 \mathrm{~W} \mathrm{~cm}^{-2}\right.$, and $5 \mathrm{~min}$ ) was applied in the PBS- and DINC-treated groups, and the cells were further cultured for another $24 \mathrm{~h}$.

Subsequently, the cells were washed, trypsinized, resuspended in PBS, stained using the Annexin V-FITC/PI apoptosis detection kit, and analyzed using FCM.

\section{Animal model}

All of the animal experiments were studied in accordance with the Guidelines for Care and Use of Laboratory Animals of Beijing Vital River Laboratory Animal Technology Co., Ltd and approved by the Institutional Animal Care and Use Committee of Beijing Vital River Laboratory Animal Technology Co., Ltd.

\section{In vivo imaging and biodistribution analysis of DINCs}

The tumor-bearing nude mice were randomly divided into 2 groups (three per group). Next, $200 \mu \mathrm{L}$ of free ICG and DINCs (containing $1 \mathrm{mg} \mathrm{mL^{-1 }}$ ICG) was injected via the tail vein, respectively. Images and FL semiquantitative analysis of ICG in vivo were performed at 1, 3, 6, 12, 24, 36 and $48 \mathrm{~h}$ after injection using an IVIS Imaging Spectrum System (PerkinElmer, USA $)$ at certain parameters $\left(\lambda_{\mathrm{ex}}=745 \mathrm{~nm}, \lambda_{\mathrm{em}}=820 \mathrm{~nm}\right.$, binning $=1$, and exposure time $=0.1 \mathrm{~s}$ ). The mice were then sacrificed, and organs including heart, liver, spleen, lungs, kidneys, brain and tumor were collected for FL imaging. After FL imaging, the tumors were isolated and immediately frozen at $-80{ }^{\circ} \mathrm{C}$. Furthermore, frozen sections of tumor were prepared for fluorescence imaging and fluorescence intensity analysis.

\section{In vivo photothermal evaluation of DINCs}

The tumor-bearing nude mice were randomly divided into 2 groups (three per group): the free ICG group and the DINC group. Two hundred microliters of free ICG and DINCs (containing $1 \mathrm{mg} \mathrm{mL}^{-1} \mathrm{ICG}$ ) was injected via the tail vein, respectively. At 0 , 24 , and $48 \mathrm{~h}$ post-administration, the tumor site was irradiated

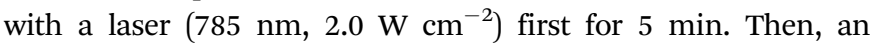
infrared thermal imager was utilized to photograph and record the temperature change of the mouse body. Two-dimensional
(2D) infrared thermographic images were processed with SmartView 3.14 software (Fluke, USA).

\section{In vivo antitumor efficacy and biosafety evaluation of DINCs}

The tumor-bearing nude mice were randomly divided into 5 groups (three per group) to evaluate the antitumor efficacy. Two hundred microliters of PBS, free DOX (containing $0.25 \mathrm{mg} \mathrm{mL}{ }^{-1} \mathrm{DOX}$ ) and DINCs (containing $0.25 \mathrm{mg} \mathrm{mL}$ DOX and $1 \mathrm{mg} \mathrm{mL}^{-1}$ ICG) were injected via the tail vein 4 times (on days 1, 8, 15 and 22). For the laser treatment groups, the tumor site was irradiated with a laser $\left(785 \mathrm{~nm}, 2 \mathrm{~W} \mathrm{~cm}^{-2}\right)$ for 5 min on days 2, 9, 16 and 23. The tumor volumes and body weight changes of the mice were recorded, and the inhibition rate of the tumor (IRT) was calculated by the following formula:

IRT $(\%)=(1-D / S) \times 100 \%(D$ : tumor volume of treatment group; $S$ : tumor volume of saline group).

After 28 days, the mice were sacrificed, and their main organs (heart, liver, spleen, lungs, and kidneys) and tumors were excised, sectioned, and stained with hematoxylin and eosin (H\&E). Each section was imaged using a digital microscope to detect histopathological changes. Furthermore, the terminal deoxynucleotidyl transferase-mediated dUTP nick end labeling (TUNEL) apoptosis assay kit was used to stain tumor slices for the evaluation of cell apoptosis by CLSM.

\section{Statistical analysis}

Data are expressed as the mean \pm standard deviation (SD). The two-tailed Student's tests or one-way ANOVA were applied for statistical evaluation.

\section{Results and discussion}

\section{Preparation and characterization of DINCs}

DOX and ICG-coloaded PEG-PPS-cRGD nanocapsules (DINCs) cross-linked by disulfide bond were synthesized using a selfcrosslinked approach of disulfide exchange reaction, which is an easy-to-accomplish and particularly meaningful strategy because it can completely avoid using multiple chemicals such as monomers, crosslinkers and initiators in traditional synthesis of polymer nanogels/nanocapsules, which may significantly improve tedious purification processes and serious toxicity of the products.

Owing to the branched structure and higher PEG densities, four-arm PEG-PPS-cRGD nanocapsules exhibited outstanding properties for holding the loaded drugs, and drug loading capacity of $4.8 \%$ and $15.9 \%$ and drug encapsulation efficiency of $49 \%$ and $90 \%$ were detected for DOX and ICG in DINCs, respectively. The DINCs exhibited an obvious core-shell structure with a dark drug core coated with a gray polymer layer, suggesting that DOX and ICG were successfully encapsulated inside the polymer nanocapsules (Fig. 2a). The TEM image also revealed that the particle size of the DINCs was in the range from 100 to $200 \mathrm{~nm}$. Consistent with the TEM result, an average diameter of approximately $152.6 \mathrm{~nm}$ was tested by DLS 
(a)

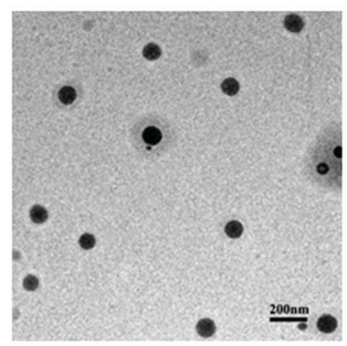

(d)

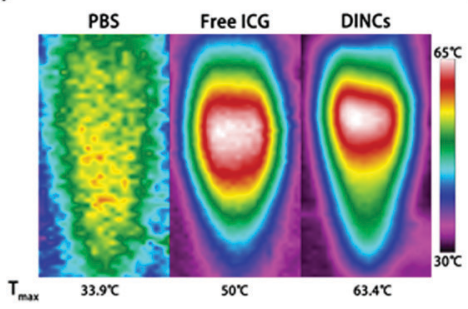

(g)

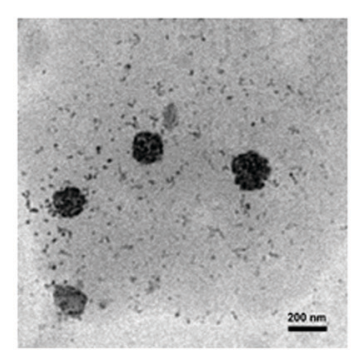

(b)

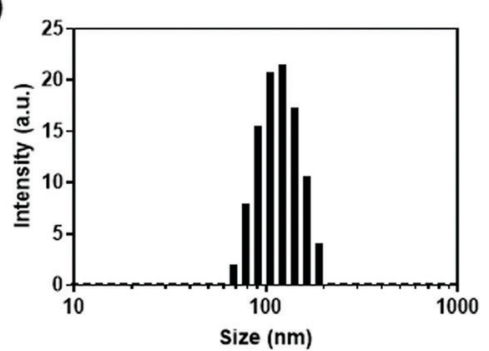

(e)

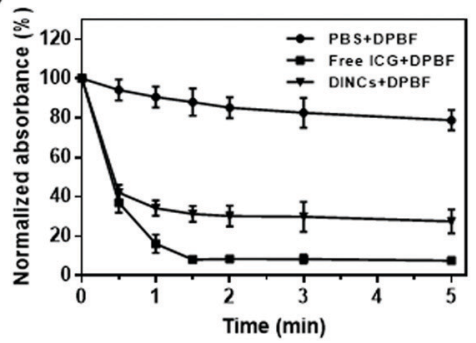

(c)

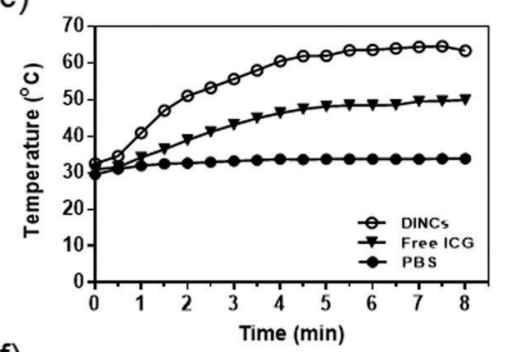

(f)

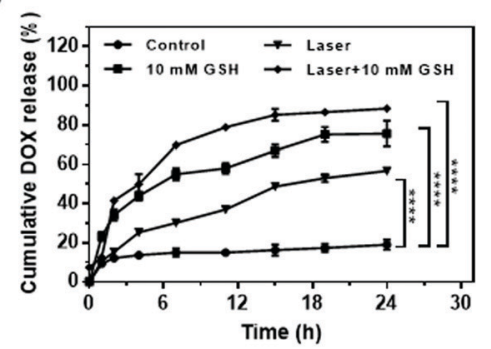

Fig. 2 Preparation and characterization of DINCs. (a) TEM image of DINCs. (b) The particle size distribution of DINCs measured by DLS. (c) The temperature-rise profiles of PBS, free ICG, and DINC solutions irradiated with a continuous laser $\left(785 \mathrm{~nm}, 2.0 \mathrm{~W} \mathrm{~cm}^{-2}\right)$. (d) Infrared thermographic maps of centrifuge tubes with PBS, free ICG, and DINC solutions irradiated with a continuous laser $\left(785 \mathrm{~nm}, 2.0 \mathrm{~W} \mathrm{~cm}^{-2}\right.$ ) for 5 min. (e) Normalized absorbance intensity of DPBF in PBS, free ICG, and DINC solutions irradiated with a continuous laser $\left(785 \mathrm{~nm}, 2.0 \mathrm{~W} \mathrm{~cm}^{-2}\right)$. Data are presented as the means \pm SD $(n=3)$. (f) DOX release profiles of DINCs with and without laser irradiation (785 nm, $2.0 \mathrm{~W} \mathrm{~cm}^{-2}$, and $5 \mathrm{~min}$ ), GSH, and laser irradiation (785 $\mathrm{nm}$, $2.0 \mathrm{~W} \mathrm{~cm}^{-2}$, and $\left.5 \mathrm{~min}\right)$ plus GSH. The data are shown as the means $\pm \mathrm{SD}(n=3)$; ${ }^{* \star \star} P<0.0001$. $(\mathrm{g})$ TEM image of DINCs irradiated with a continuous laser $\left(785 \mathrm{~nm}, 2.0 \mathrm{~W} \mathrm{~cm}^{-2}\right)$ for $5 \mathrm{~min}$.

(Fig. 2b), which confirmed that the DINCs possessed a suitable size for passive tumor-targeting through the enhanced permeability and retention effect (EPR). The zeta potential of DINCs was $-2.10 \mathrm{mV}$, indicating that the DINCs have slight negative surface potential, which would be perfect for prolonging its blood circulation time during the treatment. Furthermore, to assess aqueous phase dispersibility and stability, DINCs were dispersed in PBS buffer for long-term incubation. An average particle size of $153.4 \mathrm{~nm}$ was observed for DINCs incubated in PBS for 1 week (Fig. S1, ESI $\dagger$ ), proving that DINCs have good dispersion stability in biological media. As shown in Fig. S2 (ESI $\dagger$ ), compared with free ICG solution prepared by the solvent-antisolvent method, the DINC solution was highly transparent with no sediment events after one month, indicating that PEG-PPS-cRGD nanocapsules can carry both DOX and ICG agents with excellent stability in the aqueous phase, benefiting their application by vein injection.

To evaluate the photothermal effect of DINCs, we used a digital thermometer and infrared thermal imager to monitor temperature changes under NIR laser irradiation $(785 \mathrm{~nm}$, $2.0 \mathrm{~W} \mathrm{~cm}^{-2}$ ). As shown in Fig. $2 \mathrm{c}$, the temperature of free ICG and DINCs rapidly increased to 48.2 and $62.0^{\circ} \mathrm{C}$ during the first
5 min of irradiation and retained an equilibrium for the remaining time, while the temperature of PBS only increased to $33.9^{\circ} \mathrm{C}$. Furthermore, the highest temperatures of 33.9, 50, and $63.4{ }^{\circ} \mathrm{C}$ were observed for PBS, free ICG and DINCs using an infrared thermal imager after 5 min of irradiation (Fig. 2d). Taken together, the DINCs exhibited a higher temperature increase than free ICG under laser irradiation, suggesting that the encapsulation of ICG in polymer nanocapsules enhanced its photothermal conversion efficiency due to a more condensed concentration and slower heat dissipation process, which would benefit PTT and DOX release of DINCs. ${ }^{37}$

The ROS generation ability of DINCs was investigated by measuring the change in concentration of ${ }^{1} \mathrm{O}_{2}$ under NIR laser irradiation. DPBF, which could react with ${ }^{1} \mathrm{O}_{2}$ to induce a reduced absorbance at $410 \mathrm{~nm}$, was used to evaluate the amount of ${ }^{1} \mathrm{O}_{2}$ using a UV-visible spectrophotometer. ${ }^{38}$ As shown in Fig. 2e, the decrease in DPBF absorbance was negligible in the PBS group. However, the absorbance of DPBF in the DINC group sharply dropped to $34.3 \%$ and $27.5 \%$ of its original value with short-term $(1 \mathrm{~min})$ and long-term $(5 \mathrm{~min})$ laser irradiation, respectively, demonstrating that DINCs could respond to laser irradiation to generate ${ }^{1} \mathrm{O}_{2}$, which can be used 
not only for PDT but also for promoting DINC internalization into cells by the PCI effect.

To assess the DOX release profiles of DINCs, various triggers including GSH and NIR laser were adopted, and the cumulative release rates are shown in Fig. 2f. DINCs without any triggers released only $19 \%$ of DOX during the testing period of $24 \mathrm{~h}$. In contrast, cumulative DOX release rates of $75 \%$ and $56 \%$ were observed for GSH (10 mM) and the NIR laser (785 nm, $2.0 \mathrm{~W} \mathrm{~cm} \mathrm{~cm}^{-2}$, and $5 \mathrm{~min}$ ) treatment groups, respectively. In particular, DINCs treated with the combination of GSH and NIR laser exhibited the highest dissolution rate of approximately $88 \%$. To further demonstrate that the combination of GSH and NIR laser irradiation could synergistically promote DOX release, we used TEM to examine changes in the morphology of DINCs. As shown in Fig. $2 \mathrm{~g}$, after treatment with both GSH and NIR laser, the structure of DINCs exhibited a loose state in comparison to the initial structure, and many free small drug particles were released from the DINCs, suggesting that DOX release of DINCs could be significantly accelerated by the combination of GSH and NIR laser irradiation. Taken together, the studies of DOX release profiles confirmed that DINCs had outstanding reduction-responsive features; additionally, DOX release could also be further accelerated by NIR laser irradiation due to the activation of hyperthermia from coloaded ICG.

\section{In vitro cellular uptake of DINCs}

The cellular uptake of DINCs was investigated using CLSM and FCM. As shown in Fig. 3a, both green (DOX) and red (ICG) fluorescence signals were present and colocalized in the cytoplasm and nucleus of SCC-15 cells treated with DINCs, indicating that DOX and ICG were simultaneously transported into the cells by the PEG-PPS-cRGD nanocapsules. In particular, the cells treated with short-term laser irradiation exhibited significantly increased cellular uptake of DINCs compared with that of the cells without laser irradiation, as evidenced by the highest red and green florescence intensities, explaining that DINCs generated a PCI effect under laser irradiation. Fig. S3 $(\mathrm{ESI} \dagger)$ shows fluorescence colocalization analysis of SCC-15

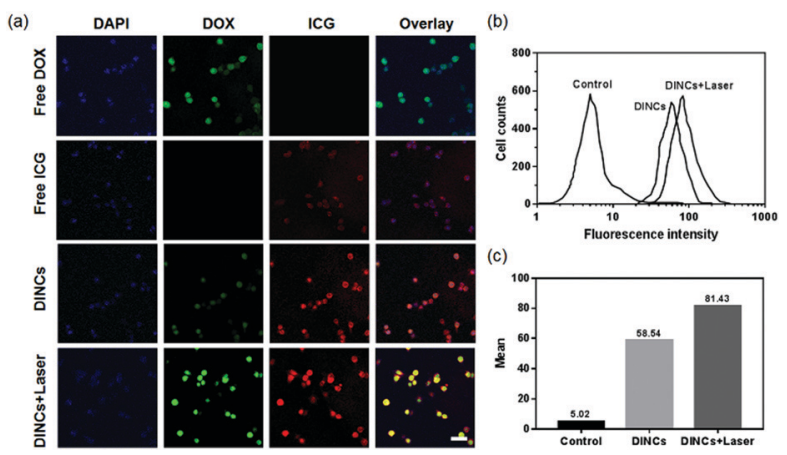

Fig. 3 In vitro cellular uptake of DINCs. (a) CLSM images of SCC-15 cells incubated with free DOX, free ICG, DINCs, and DINCs plus short-term laser irradiation (785 nm, $2.0 \mathrm{~W} \mathrm{~cm}^{-2}$, and $1 \mathrm{~min}$ ). Scale bar: $100 \mu \mathrm{m}$. (b) and (c) FCM of SCC-15 cells incubated with DINCs and DINCs plus short-term laser irradiation (785 $\mathrm{nm}, 2.0 \mathrm{~W} \mathrm{~cm}^{-2}$, and $1 \mathrm{~min}$ ) for DOX fluorescence intensity. cells treated with DINCs plus laser irradiation, and the morphological characteristics of fluorescence curves of both DOX and ICG were near coincident. Furthermore, the FCM results also validated that the fluorescence intensity was 1.39 times higher in the cells treated with DINCs in the presence compared with those in the absence of laser irradiation (Fig. 3b and c). Taken together, PEG-PPS-cRGD nanocapsules were capable of delivering both DOX and ICG into cells simultaneously, and the cellular uptake efficiency could be further enhanced by the NIR laser irradiation-induced PCI effect.

\section{Detection of intracellular ROS generation by DINCs}

Intracellular ROS generation by DINCs under laser irradiation was assessed using DCFH-DA, which can be oxidized in cells by ROS to DCF to emit bright green fluorescence. ${ }^{39}$ As shown in Fig. 4a, it was difficult to observe the green fluorescence in PBS-treated cells with laser irradiation. In free ICG-treated cells, an extremely weak fluorescence signal was detected, which was probably due to the poor intracellular uptake ability of free ICG. After laser irradiation, the cells treated with DINCs exhibited increased ROS generation compared with that of the PBS and free ICG groups, as evidenced by the highest green fluorescence intensity in the cells. The FCM results also confirmed that after laser irradiation, DINC-treated cells had the strongest fluorescence intensity of DCF, which was 8.3 and 6.9 times higher than in the PBS and free ICG-treated cells (Fig. 4b and c). Consequently, PEG-PPS-cRGD nanocapsules efficiently enhanced the intracellular uptake rate of ICG, resulting in higher concentrations of intracellular ROS after laser irradiation, which could be used for PDT.

\section{In vitro cytotoxicity detection of DINCs}

To investigate the in vitro cytotoxicity of DINCs toward SCC-15 cells, MTT assay was adopted. As shown in Fig. 5a, the PEG-PPScRGD branched copolymer presented excellent biocompatibility, as indicated by more than $85 \%$ cell survival at the highest concentration of $360 \mu \mathrm{g} \mathrm{mL} \mathrm{m}^{-1}$. Fig. $5 \mathrm{~b}$ shows the cytotoxicity of the cells treated with PBS with laser irradiation, free DOX, and DINCs with or without laser irradiation. PBS with laser

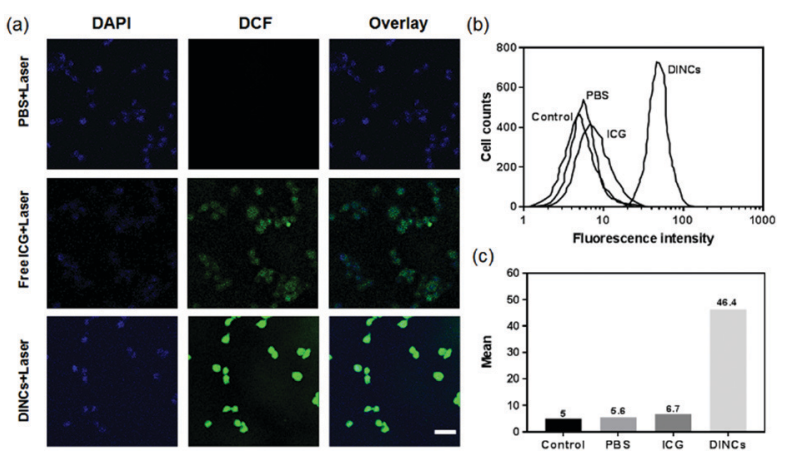

Fig. 4 Detection of intracellular ROS generation of DINCs. (a) CLSM images of SCC-15 cells incubated with PBS, free ICG, and DINCs under laser irradiation ( $785 \mathrm{~nm}, 2.0 \mathrm{~W} \mathrm{~cm}^{-2}$, and $\left.1 \mathrm{~min}\right)$. Scale bar: $100 \mu \mathrm{m}$. (b) and (c) FCM of SCC -15 cells incubated with PBS, free ICG, and DINCs with laser irradiation (785 nm, $2.0 \mathrm{~W} \mathrm{~cm}^{-2}$, and $1 \mathrm{~min}$ ) for DCF fluorescence intensity. 
(a)

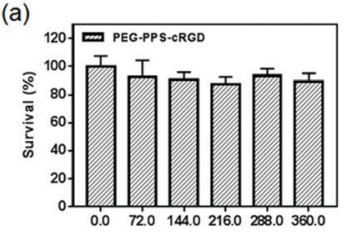

(b) Polymer concentration (ug/ml)
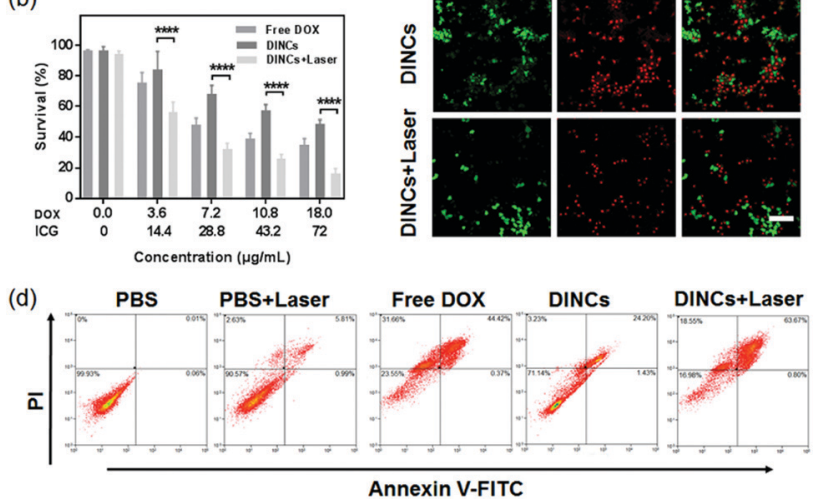

Fig. 5 In vitro cytotoxicity detection of DINCs. (a) MTT assay of SCC-15 cells treated with PEG-PPS-CRGD branched copolymers. (b) MTT assay of SCC -15 cells treated with free DOX, DINCs, and DINCs plus laser irradiation (785 nm, $2.0 \mathrm{~W} \mathrm{~cm}^{-2}$, and $5 \mathrm{~min}$ ). Data are presented as the means $\pm \mathrm{SD}$ ( $n=3$ ); $* * * * P<0.0001$. (c) Live-dead staining (green $=$ live, and red $=$ dead) of SCC-15 cells treated with PBS plus laser irradiation, free DOX, DINCs, and DINCs plus laser irradiation. Scale bar: $100 \mu \mathrm{m}$. (d) Apoptosis analysis of SCC -15 cells treated with PBS, PBS plus laser irradiation, free DOX, DINCs, and DINCs plus laser irradiation by Annexin-V/PI staining

irradiation did not influence cell viability, indicating that laser irradiation at a power density of $2.0 \mathrm{~W} \mathrm{~cm}^{-2}$ was appropriate for the treatment of SCC-15 cells. Of note, DINC-treated cells showed drug concentration-related cytotoxicity, with a gradual decline in cell viability with increasing drug concentration. More importantly, DINC-treated cells with laser irradiation showed $84.5 \%$ cell death at the highest drug concentration $\left(18 \mu \mathrm{g} \mathrm{mL}^{-1}\right.$ of DOX and $72 \mu \mathrm{g} \mathrm{mL} \mathrm{m}^{-1}$ of ICG), demonstrating a 1.96-fold increase in cytotoxicity compared with the $43.2 \%$ observed for DINC-treated cells without laser irradiation. These results demonstrated that laser irradiation significantly enhanced the cytotoxicity of DINCs by triggering a synergistic photo-chemo combined activity.

The live-dead assay was performed to visually observe the cytotoxicity of DINCs, and the fluorescence images are shown in Fig. 5c. Consistent with the MTT results, the PBS-treated cells with laser irradiation did not show obvious cell death, with only a few cells exhibiting red fluorescence. The cells incubated with DINCs exhibited effective cytotoxicity, as demonstrated by a greater number of cells emitting red fluorescence. Moreover, after laser irradiation, the cytotoxicity of DINC-treated cells was further enhanced, as evidenced by the significantly reduced cell density and the highest proportions of red cells, suggesting that the NIR light-triggered photo-chemo combination therapy of DINCs was able to achieve more cell death.

Furthermore, Annexin-V/PI double-staining assay was used to evaluate apoptotic or necrotic cells, and the results were quantified by FCM, as shown in Fig. 5 d. The percentage of total apoptotic cells (including early and late apoptosis) treated with DINCs with laser dramatically increased to $64.5 \%$, which was much higher compared with the cells treated with PBS $(0.07 \%)$, PBS with laser $(6.8 \%)$, free DOX $(44.8 \%)$, and DINCs without laser $(25.6 \%)$. These results demonstrated that the apoptosisinducing ability of DINCs could be sharply enhanced in the presence of laser irradiation.

\section{In vivo imaging and biodistribution of DINCs}

To evaluate the tumor-targeting ability, the BALB/c nude SCC15-tumor-bearing mice were intravenously injected with free ICG and DINCs, and the fluorescence distribution of ICG was subsequently recorded at different time points $(0,1,3,6,12,24$, 36 , and $48 \mathrm{~h}$ ) using an ex/in vivo imaging system. As shown in Fig. 6a, fluorescence signals were detected throughout the body of the mice within the first $6 \mathrm{~h}$ in both the free ICG and DINC treatment groups. Subsequently, with extended time, the fluorescence intensity at the tumor site was significantly enhanced in DINC-treated mice compared with free ICGtreated mice at every time point. However, the fluorescence signal of free ICG was barely detected at $48 \mathrm{~h}$ post-injection. At $48 \mathrm{~h}$ post-administration, the mice were sacrificed, and various main organs and tumor were isolated for ex vivo imaging for further detection of the fluorescence distribution of free ICG and DINCs. In contrast to free ICG, a higher fluorescence signal was detected on the tumor of DINC-treated mice (Fig. 6b), and the quantitative average fluorescence intensity revealed a 1.9-fold increase (Fig. 6c). Furthermore, the fluorescence images of tumor sections confirmed that DINCs were capable of penetrating deep into the tumor tissue (Fig. 6d), and the fluorescence intensity of ICG in the DINC-treated group was much higher than that of the group treated with free ICG (Fig. S4, ESI $\dagger$ ). Overall, these biodistribution results validated that ICG loading into PEG-PPS-cRGD nanocapsules significantly enhanced its tumor-targeting ability through both the EPR effect and cRGD-integrin binding capability, resulting in higher tumor accumulation. ${ }^{40,41}$

\section{In vivo photothermal effect of DINCs}

The SCC-15 tumor-bearing mice were intravenously injected with free ICG (10 $\mathrm{mg} \mathrm{kg}^{-1}$ ICG) and DINCs $\left(2.5 \mathrm{mg} \mathrm{kg}^{-1}\right.$ DOX and $10 \mathrm{mg} \mathrm{kg}^{-1}$ ICG), and the photothermal effect on the tumor site after laser irradiation ( $785 \mathrm{~nm}, 2 \mathrm{~W} \mathrm{~cm}^{-2}$, and $5 \mathrm{~min}$ ) was recorded using an infrared thermal imager (Fig. 6e). For the mice treated with free ICG, temperatures of 30.3, 44.7 and $40.6{ }^{\circ} \mathrm{C}$ were observed at 0,24 and $48 \mathrm{~h}$ post-administration, respectively. Of note, the tumors treated with DINCs showed an enhanced photothermal effect compared with those that received free ICG, as indicated by the temperature increase to above $50{ }^{\circ} \mathrm{C}$ at both studied time points, suggesting that DINCs could generate a higher temperature at the tumor site after laser irradiation due to its outstanding tumor-targeting ability, leading to higher tumor accumulation. Moreover, the results demonstrated that DINCs with NIR laser could achieve an efficient photothermal effect that would be capable of irreversibly destroying the tumor. 
(a)

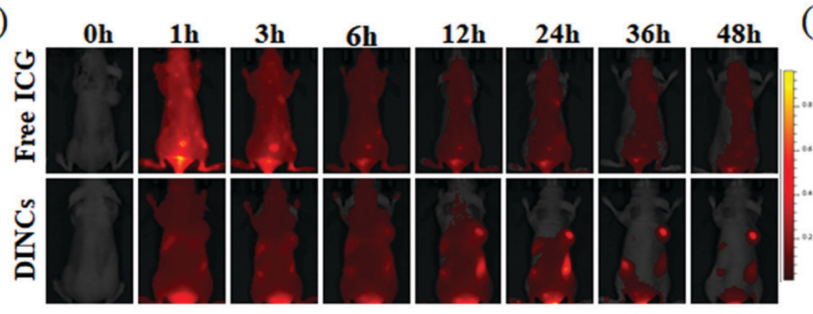

(c)

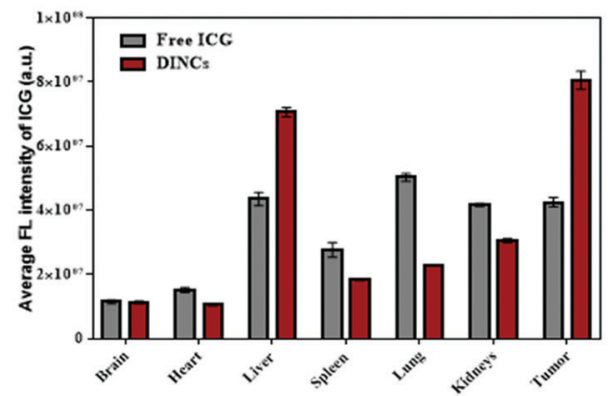

(e)
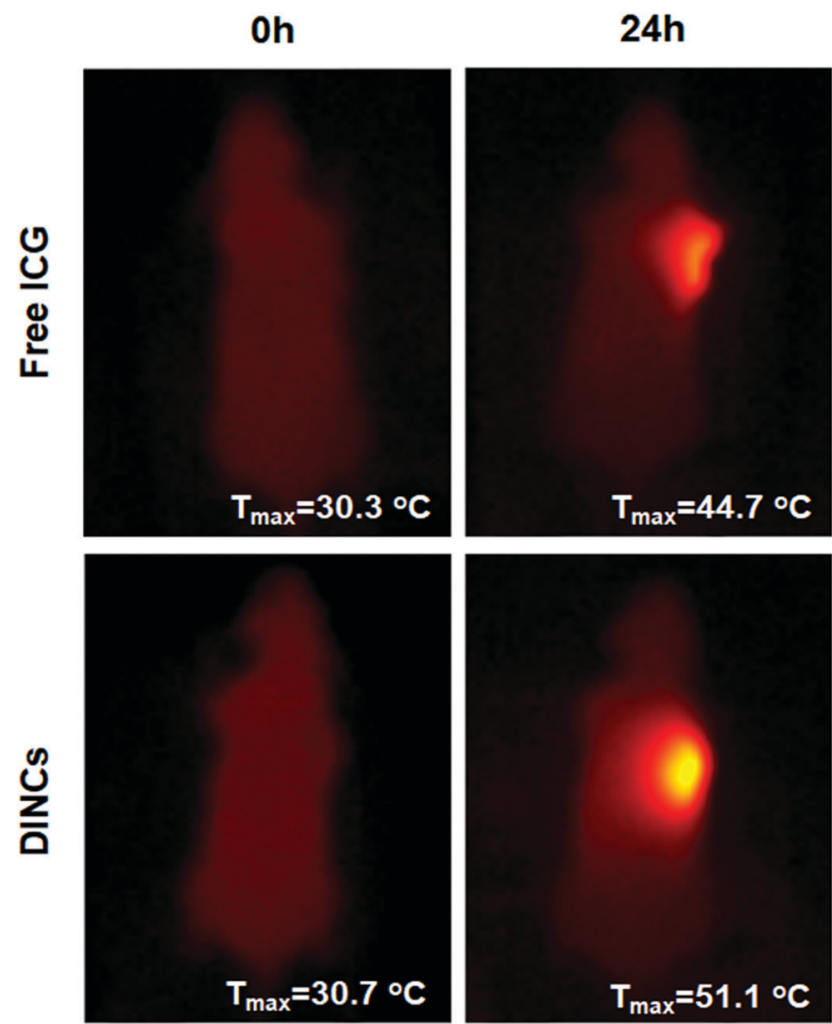

(b)

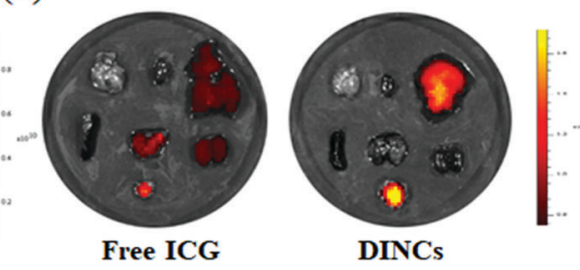

(d)

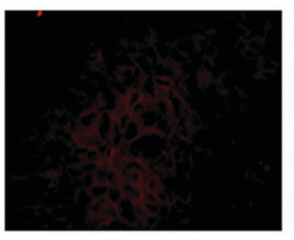

Free ICG

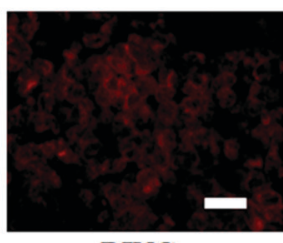

DINCs
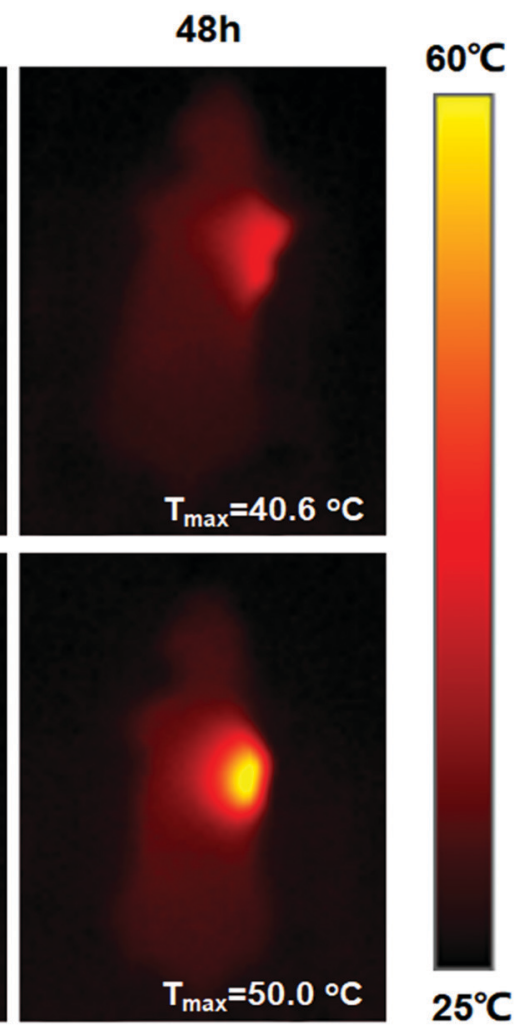

Fig. 6 In vivo imaging and biodistribution of DINCs. (a) In vivo fluorescence imaging of nude mice bearing SCC-15 tumors after intravenous injection with free ICG and DINCs (10 $\mathrm{mg} \mathrm{kg}^{-1} \mathrm{ICG}$ ) at different times. (b) Ex vivo fluorescence imaging of various organs and tumor tissue after intravenous injection with free ICG and DINCs at $48 \mathrm{~h}$. (c) Average fluorescence intensity of the organs and tumor by quantitative analysis at $48 \mathrm{~h}$ post-injection. Data represent means $\pm S D(n=3)$. (d) ICG distribution within the tumor tissue at $48 \mathrm{~h}$ post-injection. Scale bar: $100 \mu \mathrm{m}$. (e) Infrared thermographic images of nude mice bearing SCC-15 tumors after intravenous injection with free ICG and DINCs $\left(10 \mathrm{mg} \mathrm{kg}^{-1} \mathrm{ICG}\right)$ at different times; the tumor site was exposed under laser irradiation $\left(785 \mathrm{~nm}, 2.0 \mathrm{~W} \mathrm{~cm}^{-2}\right)$ for 5 min before taking the pictures.

\section{In vivo photo-chemo combination effect of DINCs}

The in vivo photo-chemo combination effect of DINCs in the treatment of squamous cell carcinoma was investigated using SCC-15 tumor-bearing mice. As shown in Fig. 7a-c, the DINC treatment group exhibited stronger tumor growth inhibition compared with the PBS and free DOX treatment groups, with an inhibition rate of $72.6 \%$ at day 28 , demonstrating that the tumortargeting ability of PEG-PPS-cRGD nanocapsules could effectively improve the effect of anticancer drugs. Additionally, the tumors treated with PBS plus laser irradiation $\left(785 \mathrm{~nm}, 2 \mathrm{~W} \mathrm{~cm}^{-2}\right.$, 
and $5 \mathrm{~min}$ ) grew rapidly, demonstrating that tumor growth was not inhibited by laser irradiation under these treatment conditions. In particular, the tumors treated with DINCs with laser irradiation had completely disappeared at day 24 , and no tumor regrowth was observed even at day 49 (Fig. S5, ESI $\dagger$ ), suggesting that NIR laser-triggered photo-chemo combination therapy of DINCs could effectively kill squamous cell carcinoma tumors with no recurrence. To further assess the antitumor effect of DINCs, H\&E and TUNEL staining of tumor sections are shown in Fig. 7d. In comparison to the PBS and free DOX groups, the DINC group showed more cells with nuclear condensation and fragmentation in the H\&E images, demonstrating that DINCs had better antitumor activity. Additionally, the tumors treated with DINCs showed more apoptotic cells than those in PBS and free DOX groups, as evidenced by more green fluorescent cells in the tumor section stained using the TUNEL assay, validating the outstanding tumor growth inhibition ability of DINCs. Overall, the DINCs showed prominent tumor inhibition efficacy, especially in NIR laser-triggered photo-chemo combination therapy.

\section{Biosafety evaluation of DINCs}

The in vivo toxicity of DINCs was evaluated by recording body weight changes and by histological analysis of main organs. As shown in Fig. S6 (ESI $\dagger$ ), there was no obvious body weight loss during the treatment with DINCs with or without laser irradiation. Furthermore, histological analysis of H\&E stained sections showed that the mice treated by DINCs with or without laser irradiation had no anomalous histopathological changes in the tested organs, including brain, heart, liver, spleen, lungs, and kidneys (Fig. S7, ESI $\dagger$ ). These results confirmed that the treatment with DINCs with or without laser irradiation would not cause significant toxic side effects in vivo. (a)

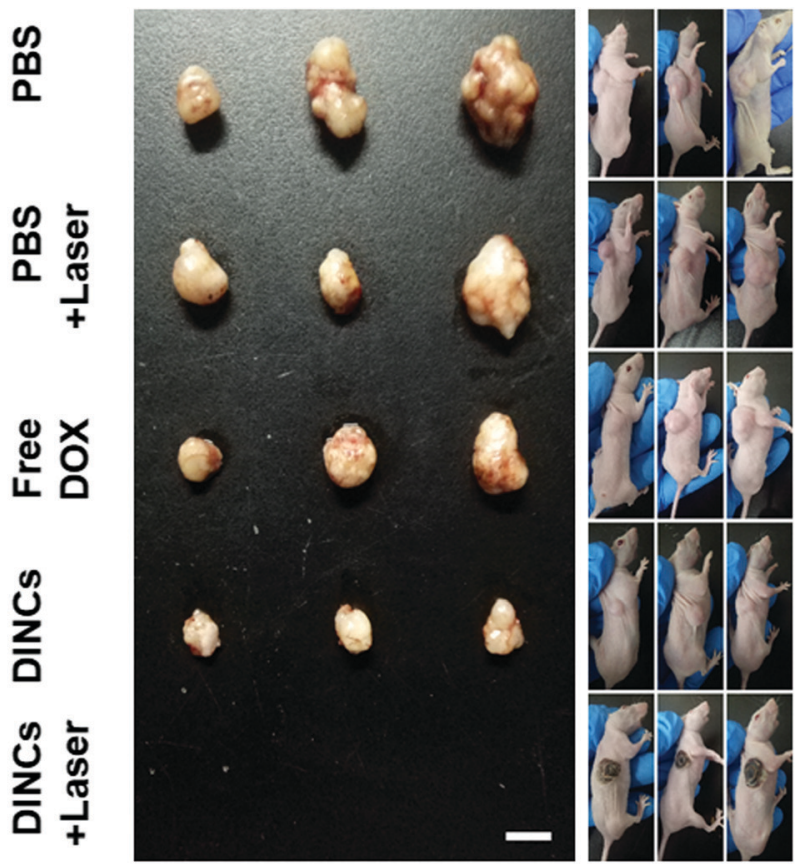

(d)
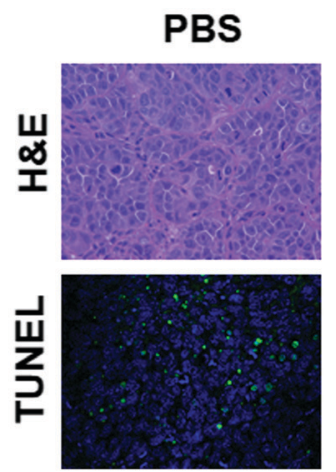

PBS+Laser
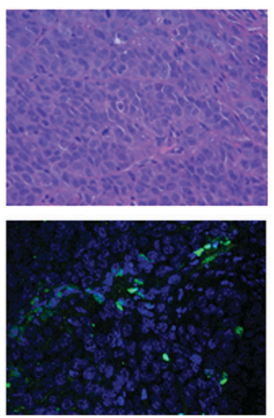
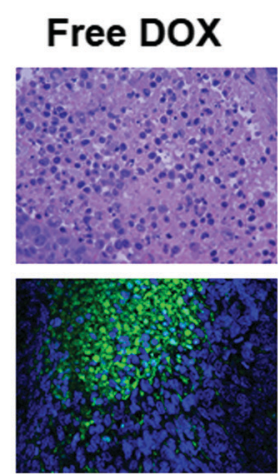

(b)

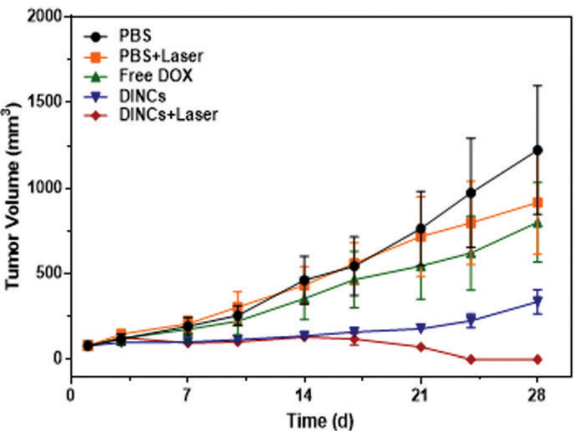

(c)

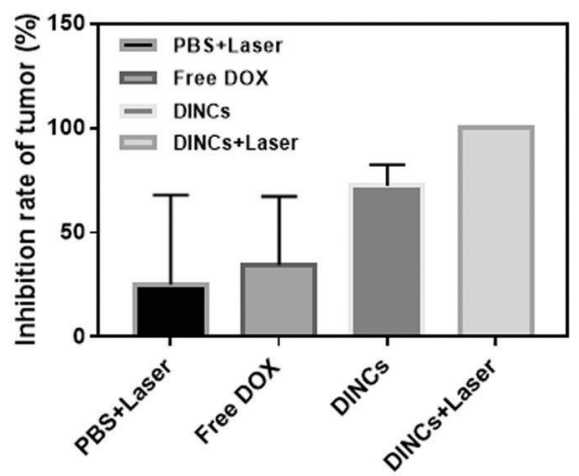

DINCs

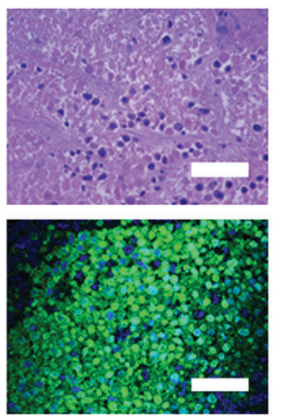

DINCs+Laser

No tumor

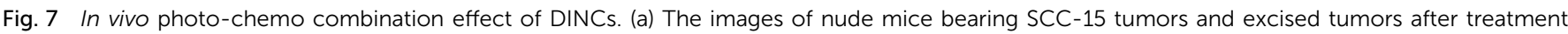

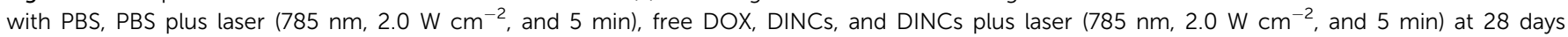

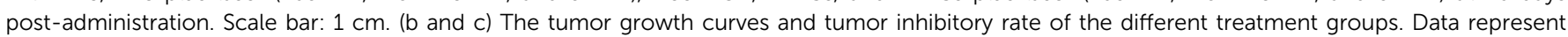
means $\pm \mathrm{SD}(n=3) ;{ }^{* \star \star \star} P<0.0001$. (d) H\&E and TUNEL staining of the tumor tissues of the different treatment groups. Scale bar: $100 \mu \mathrm{m}$. 


\section{Conclusions}

In summary, we developed an NIR light-triggered synergistic photo-chemo combination therapy platform by combination drug codelivery of DOX and ICG via reduction-responsive selfcrosslinked PEG-PPS-cRGD nanocapsules. The dissolution studies demonstrated that the nanocapsules had sensitive reduction-responsive drug release profiles, while the drug release rate could be further accelerated with the assistance of NIR laser irradiation. Owing to the small size, targeting ligand of cRGD and PCI effect, the nanocapsules could effectively be taken up by squamous cells in vitro as well as target the tumor of squamous cell carcinoma in vivo. Additionally, the nanocapsules exhibited an outstanding photothermal effect and ROS generation under laser irradiation, which was also evidenced by the enhanced cytotoxicity in the treated squamous cells. Finally, the in vivo antitumor and biosafety investigations validated that this synergistic photo-chemo combination treatment by DINCs plus NIR laser irradiation could completely eliminate the tumor and significantly prevent tumor recurrence, with a favorable biological safety profile in the SCC-15 tumor-bearing mice model. Altogether, this high-performance NIR-triggered photo-chemo combination therapeutic platform may offer a promising strategy for the treatment of various malignant tumors.

\section{Conflicts of interest}

There are no conflicts to declare.

\section{Acknowledgements}

This work was financially supported by the National Natural Science Foundation of China (Grant 52072023, 21622601, and 51972339), and the Open Project Program of the State Key Laboratory of Organic-Inorganic Composites, Beijing University of Chemical Technology (Grant oic-201901010).

\section{References}

1 J. Conde, N. Oliva, Y. Zhang and N. Artzi, Nat. Mater., 2016, 15, 1128.

2 Y. Yang, W. Zhu, Z. Dong, Y. Chao, L. Xu, M. Chen and Z. Liu, Adv. Mater., 2017, 29, 1703588.

3 R. Jin, Z. Liu, Y. Bai, Y. Zhou, J. J. Gooding and X. Chen, Adv. Funct. Mater., 2018, 28, 1801961.

4 H. Zhu, H. Wang, B. Shi, L. Shangguan, W. Tong, G. Yu, Z. Mao and F. Huang, Nat. Commun., 2019, 10, 2412.

5 Y. Huang, F. Qiu, L. Shen, D. Chen, Y. Su, C. Yang, B. Li, D. Yan and X. Zhu, ACS Nano, 2016, 10, 10489-10499.

6 R. Liu, M. Yu, X. Yang, C. S. Umeshappa, C. Hu, W. Yu, L. Qin, Y. Huang and H. Gao, Adv. Funct. Mater., 2019, 29, 1808462.

7 Y. Wen, W. Zhang, N. Gong, Y.-F. Wang, H.-B. Guo, W. Guo, P. C. Wang and X.-J. Liang, Nanoscale, 2017, 9, 14347-14356.

8 W. Chen, K. Zeng, H. Liu, J. Ouyang, L. Wang, Y. Liu, H. Wang, L. Deng and Y.-N. Liu, Adv. Funct. Mater., 2017, 27, 1605795.
9 R. Zhao, G. Zheng, L. Fan, Z. Shen, K. Jiang, Y. Guo and J.-W. Shao, Acta Biomater., 2018, 70, 197-210.

10 R. Chen, J. Zhang, Y. Wang, X. Chen, J. A. Zapien and C.-S. Lee, Nanoscale, 2015, 7, 17299-17305.

11 Y. Wang, G. Wei, X. Zhang, F. Xu, X. Xiong and S. Zhou, Adv. Mater., 2017, 29, 1605357.

12 K. Zhu, G. Liu, J. Hu and S. Liu, Biomacromolecules, 2017, 18, 2571-2582.

13 T. A. Theodossiou, A. R. Gonçalves, K. Yannakopoulou, E. Skarpen and K. Berg, Angew. Chem., Int. Ed., 2015, 54, 4885-4889.

14 M. S. Mathews, V. Vo, E.-C. Shih, G. Zamora, C.-H. Sun, S. J. Madsen and H. Hirschberg, J. Environ. Pathol., Toxicol. Oncol., 2012, 31, 49-59.

15 Z. Yang, W. Fan, W. Tang, Z. Shen, Y. Dai, J. Song, Z. Wang, Y. Liu, L. Lin, L. Shan, Y. Liu, O. Jacobson, P. Rong, W. Wang and X. Chen, Angew. Chem., 2018, 130, 14297-14301.

16 Y. Li, Y. Zhang and W. Wang, Nano Res., 2018, 11, 5424-5438. 17 H.-J. Li, J.-Z. Du, J. Liu, X.-J. Du, S. Shen, Y.-H. Zhu, X. Wang, X. Ye, S. Nie and J. Wang, ACS Nano, 2016, 10, 6753-6761.

18 H. Han, D. Valdepérez, Q. Jin, B. Yang, Z. Li, Y. Wu, B. Pelaz, W. J. Parak and J. Ji, ACS Nano, 2017, 11, 1281-1291.

19 D. Chen, G. Zhang, R. Li, M. Guan, X. Wang, T. Zou, Y. Zhang, C. Wang, C. Shu, H. Hong and L.-J. Wan, J. Am. Chem. Soc., 2018, 140, 7373-7376.

20 S. Mura, J. Nicolas and P. Couvreur, Nat. Mater., 2013, 12, 991.

21 R. Cheng, F. Meng, C. Deng, H.-A. Klok and Z. Zhong, Biomaterials, 2013, 34, 3647-3657.

22 H. Kim, S. Kim, C. Park, H. Lee, H. J. Park and C. Kim, Adv. Mater., 2010, 22, 4280-4283.

23 M. Zhao, A. Biswas, B. Hu, K.-I. Joo, P. Wang, Z. Gu and Y. Tang, Biomaterials, 2011, 32, 5223-5230.

24 J. Li, M. Huo, J. Wang, J. Zhou, J. M. Mohammad, Y. Zhang, Q. Zhu, A. Y. Waddad and Q. Zhang, Biomaterials, 2012, 33, 2310-2320.

25 D. Zhong, Z. Tu, X. Zhang, Y. Li, X. Xu and Z. Gu, Biomacromolecules, 2017, 18, 3498-3505.

26 M. Li, Y. Gao, Y. Yuan, Y. Wu, Z. Song, B. Z. Tang, B. Liu and Q. C. Zheng, ACS Nano, 2017, 11, 3922-3932.

27 S. Zhang, C. Sun, J. Zeng, Q. Sun, G. Wang, Y. Wang, Y. Wu, S. Dou, M. Gao and Z. Li, Adv. Mater., 2016, 28, 8927-8936.

28 X. He, X. Bao, H. Cao, Z. Zhang, Q. Yin, W. Gu, L. Chen, H. Yu and Y. Li, Adv. Funct. Mater., 2015, 25, 2831-2839.

29 L. Zhu, F. Perche, T. Wang and V. P. Torchilin, Biomaterials, 2014, 35, 4213-4222.

30 G. Saravanakumar, J. Lee, J. Kim and W. J. Kim, Chem. Commun., 2015, 51, 9995-9998.

31 W. Liu, M. Ruan, Y. Wang, R. Song, X. Ji, J. Xu, J. Dai and W. Xue, Small, 2018, 14, 1801754.

32 R. Vankayala and K. C. Hwang, Adv. Mater., 2018, 30, 1706320.

33 H. Ding, K.-T. Yong, I. Roy, R. Hu, F. Wu, L. Zhao, W.-C. Law, W. Zhao, W. Ji, L. Liu, E. J. Bergey and P. N. Prasad, Nanotechnology, 2011, 22, 165101. 
34 J. Tao, Z. Tan, L. Diao, Z. Ji, J. Zhu, W. Chen and Y. Hu, RSC Adv., 2018, 8, 21735-21744.

35 Y.-C. Su, P.-A. Burnouf, K.-H. Chuang, B.-M. Chen, T.-L. Cheng and S. R. Roffler, Nat. Commun., 2017, 8, 15507.

36 J. Zhang, Y. Li, J. Wang, S. Qi, X. Song, C. Tao, Y. Le, N. Wen and J. Chen, RSC Adv., 2017, 7, 53552-53562.

37 M. Zheng, C. Yue, Y. Ma, P. Gong, P. Zhao, C. Zheng, Z. Sheng, P. Zhang, Z. Wang and L. Cai, ACS Nano, 2013, 7, 2056-2067.
38 D. Wöhrle, M. Shopova, S. Müller, A. D. Milev, V. N. Mantareva and K. K. Krastev, J. Photochem. Photobiol., $B, 1993,21,155-165$.

39 V. G. Deepagan, S. Kwon, D. G. You, V. Q. Nguyen, W. Um, H. Ko, H. Lee, D.-G. Jo, Y. M. Kang and J. H. Park, Biomaterials, 2016, 103, 56-66.

40 S. Raha, T. Paunesku and G. Woloschak, Wiley Interdiscip. Rev.: Nanomed. Nanobiotechnol., 2011, 3, 269-281.

41 C. Zhan, B. Gu, C. Xie, J. Li, Y. Liu and W. Lu, J. Controlled Release, 2010, 143, 136-142. 